\title{
L'Homme et la Nature : en quête/enquête sensible
}

\author{
Stéphanie Chanvallon, Stéphane Héas
}

Sociologues, Laboratoire Violences, Identités, Politiques et Sports (VIPS), Université européenne de Bretagne, 35044 Rennes, France

Les articles publiés dans NSS traitent généralement des rapports matériels vitaux que les hommes ont avec la nature, car c'est à travers eux que se jouent les enjeux majeurs de leur existence sur la planète. Mais c'est en oublier les dimensions symboliques, psychologiques et, plus globalement, anthropologiques, pourtant essentielles elles aussi. D'où l'intérêt de cet article qui explore la façon dont est vécue une relation privilégiée recherchée pour elle-même avec les éléments naturels, et qui en propose une interprétation. Nous le publions pour ouvrir un débat sur ces dimensions de l'expérience humaine de la nature, car elles constituent un arrière-plan généralement occulté des appréciations sur les usages qui sont faits des « ressources » naturelles.

La Rédaction

\section{Mots-clés :}

relations Homme-

Nature ; sensations ;

sociologie ;

anthropologie ;

enquête qualitative
Résumé - Les relations de l'Homme à la Nature sont appréhendées à partir des expériences extraordinaires (rencontres des grands mammifères marins, par exemple) de passionnés doublés de professionnels de la Nature (22 récits de vie réalisés). Suscitant les sens corporels et l'émotionnel, le cognitif et le spirituel, jusqu'à l'irrationnel, ces relations se transmutent en opérateur essentiel de leur vie, combinant connaissances scientifiques et profanes, imaginaires et réalités. Les réflexions et transformations qu'elles impliquent interrogent plus largement une écologie à hauteur d'Homme.

\section{Keywords:}

\section{Man-Nature}

relationships; senses; sociology; anthropology; qualitative surveys

\begin{abstract}
Man and Nature: senses and sensitivities. We analyse Man-Nature relationships taking 22 life narratives of exceptional experiences, such as encounters with large marine mammals, by nature lovers and professionals. In arousing the physical and emotional as well as the cognitive and spiritual senses and touching even on the irrational in humans, these relations become a vital operator in their lives in combining scientific and profane knowledge, fantasies and realities. The reflections and the changes they involve raise wider questions regarding an ecology at the human level.
\end{abstract}

Les questions liées à l'écologie et au développement durable atteignent ces dernières années un pic d'intérêts et d'investissements tant au niveau national que planétaire. Elles engagent désormais chacun de nous à s'interroger sur son rapport à la Nature ${ }^{1}$ en général, et sur

Auteur correspondant: S. Chanvallon, stephanie-chanvallon@netcourrier.com

1 Nous choisissons d'écrire la Nature avec une majuscule lorsqu'elle représente l'organique comme le minéral, le microcosme comme le macrocosme, ce dont l'être humain fait partie, dans une dimension holistique. Nous faisons de même pour l'Homme quand nous en parlons en termes génériques. Nos analyses concernent cependant uniquement l'homme occidental contemporain, et plus précisément les personnes enquêtées. l'impact qu'il a sur elle, à la fois comme individu et comme membre de l'espèce humaine.

Pourtant, au moins dans les sociétés modernes, industrialisées et fortement dépendantes des nouvelles technologies de l'information et de la communication (NTIC), la plupart des individus n'ont plus de rapports avec elle qu'épisodiquement et d'une manière indirecte, quasi artificielle. Des voies (tourisme vert, " sports de nature »...) sont explorées pour retrouver le contact (Béteille, 1996 ; Corneloup, 2007 ; Gillet et Sorzana, 2008). Il n'en demeure pas moins qu'il devient difficile de percevoir comment elle est vécue, notamment sur le plan individuel. Si, pour Lussault (2003), «la société "invente" [...] ses natures acceptables et [si] en retour cette invention contribue à la configurer et à l'organiser ", cela vaut aussi pour l'individu; et ce d'autant plus qu'aujourd'hui la mobilité des 
personnes, la diffusion et le partage des connaissances permettent d'aller vivre des expériences dans un ailleurs. Que se passe-t-il alors au cœur de ces expériences? Les effets produits concernent l'individu pris dans toutes ses dimensions, du sensoriel au psychique, du sensible au transcendant, jusqu'à l'irrationnel. Un rapport privilégié avec la Nature non seulement initie à une autre " façon » de la voir (Gould, 2001), mais surtout constitue une rencontre avec soi-même (Ormiston, 2003), un retour sur soi bénéfique. Il permet dans certains cas une véritable transformation de soi.

Dans les sociétés occidentales, les relations de l'Homme à la Nature sont vécues sur un mode paradoxal. Elles s'inscrivent dans le double registre contradictoire de l'amour et de l'exploitation destructrice (Fromm, 1975 ; Wieviorka, 2007). Elles sont au cœur d'une réflexion opposant deux visions antagonistes : celle de l'Homme, être supérieur et maître de la Nature ; celle, au contraire, del'Homme partie intégrante de celle-ci en tant qu'espèce animale et devant vivre en étroite symbiose avec elle. Longtemps dominante dans les sociétés industrielles, la première de ces conceptions est battue en brèche non seulement en raison de ses conséquences sur les ressources et les milieux naturels, mais aussi en raison d'un changement profond dans les représentations respectives de l'Homme et de l'animal, au point que les frontières entre ces deux catégories deviennent de plus en plus floues: l'altérité demeure, mais elle fait question, elle prend la forme d'un défi à relever ; et il est clair qu'elle doit se penser avec et/ou au travers de l'animalité. C'est sur ce point que nous voudrions apporter une contribution.

Les relations de l'Homme aux autres espèces animales sont spécifiquement le sujet de l'anthropozoologie. Mais nombre d'autres disciplines (comme la paléoanthropologie, la neuropsychologie) fournissent, chacune à leur manière, une contribution à la compréhension de ces relations. Leur complexité appelle un travail pluridisciplinaire. Nous nous proposons de les analyser à travers une démarche de socioanthropologie qualitative, interprétative, et pour tout dire engagée, qui, à sa façon, traverse et subsume les approches disciplinaires. Elle consiste à identifier la façon particulière qu'ont certaines personnes d'appréhender la Nature dans la mesure où elles établissent avec elle une relation privilégiée. Ces personnes sont caractérisées par leurs choix, leurs parcours personnels, leurs expériences professionnelles; ce qui leur donne accès à une dimension de perception qui transgresse les frontières entre leurs personnalités et le monde naturel dans lequel elles évoluent - au point d'avoir le sentiment d'être au contact d'une Nature en soi, de «la » Nature. Nous insistons sur le fait qu'il ne s'agit ici ni d'une vision idéalisée ni d'une vision idyllique, mais de l'expression d'un sentiment éprouvé par des hommes et des femmes rencontrés plusieurs fois : la Nature vécue comme source de réalisation et de découvertes. Partant de ce travail d'identification, nous tentons de mieux comprendre l'étendue de ce sentiment en nous appuyant aussi sur la littérature, scientifique ou non ${ }^{2}$.

\section{Pour une socioanthropologie qualitative et réflexive : de l'engagement du chercheur auprès d'informateurs privilégiés}

Ce travail procède de rencontres avec des professionnels et des passionnés de nature. Ils sont par exemple biologiste, cinéaste, ethnologue, pêcheur professionnel, sportif de haut niveau, plongeur amateur, etc. Le corpus est analysé avec l'outil des récits autobiographiques. Les sources de l'enquête visent à rendre compte d'expériences variées. Les personnes choisies l'ont été parce qu'elles étaient susceptibles de « fournir les informations importantes qui ne [pouvaient] pas être aussi bien obtenues en suivant d'autres choix » (Maxwell, 1999, p. 128). Nous cherchons, à travers ces choix délibérés, à approcher des femmes et des hommes dans leur unité, dans leur part de singularité (Cuche, 2004 ; Héas, 2010). Dans cette perspective, nous avons travaillé plus sur les mots et leurs résonances, notamment affectives, que sur le nombre d'entretiens ou la fréquence des vocables utilisés. Nous nous sommes attachés à déceler, dans le penchant marqué de chaque personne pour la Nature, les éléments de compréhension identitaire, parfois intimes, de leur quête personnelle et professionnelle. C'est pourquoi, au terme d'enquête, nous préférons celui "d'en quête » (d'où le titre donné à ce texte), n'oubliant pas ainsi la possibilité que chaque personne rencontrée soit aussi « en quête de » quelque chose d'indicible, de personnel, etc. Concernant l'enquêté, nous optons aussi volontiers pour le terme de donneur d'informations, soulignant ainsi le don effectué, lequel exige une réciprocité - soit un contre-don de la part de l'enquêteur, sur le modèle de Mauss (2007) -, afin d'établir une relation confiante et sensible. Les précisions sur ces mots reflètent précisément l'optique méthodologique empruntée.

Les discours collectés, puis analysés, renvoient à des situations variées dans l'espace et le temps, leur mise à plat révèle nombre de rapprochements possibles, difficiles à percevoir autrement. Les "stratégies identitaires » des enquêtés se sont construites sur un ensemble d'éléments: enfance, relations familiales, parcours

\footnotetext{
2 Cet article ne met en avant qu'une partie de notre recherche (thèse de sociologie, réalisée dans le cadre du LARES, membre du Laboratoire d'anthropologie et de sociologie [LAS], EA2241, Université Rennes 2), dont l'intitulé exact est Anthropologie des relations de l'Homme à la Nature : la Nature vécue entre peur destructrice et communion intime. Les thèmes de la «peur", de la «domination » et de la «destruction » ne seront donc pas évoqués, même s'ils font indéniablement partie de la compréhension des relations entre l'Homme et l'animal sauvage.
} 
professionnel, expériences hors du commun, engagements novateurs. Elles leur permettent, à un moment de leur vie, de donner du sens à leur vécu, de solliciter des capacités d'adaptation dans des contextes parfois difficiles. Se rapporter à leurs fondements permet de les dévoiler et de comprendre le pourquoi et le comment de leur apparition à un moment donné.

L'approche théorique privilégiée pour mener ces analyses combine la Grounded Theory d'A. Strauss, déjà empruntée pour d'autres terrains (Héas, 2004 ; Héas et al., 2006 ; Le Hénaff et Héas, 2007 ; Lebreton et Héas, 2007 ; Lebreton et al., 2008), et la sensibilité ethnologique et épistémologique de Geertz (1986) - d'où l'intérêt que nous portons au «cycle de vie» des enquêtés ${ }^{3}$. Notre étude interprétative supposait une participation active de leur part. Afin d'éviter tout risque de déformation, nos analyses étaient mises à leur disposition. Ce souci d'attester de l'honnêteté de la démarche (Rossi et al., 2008) rejoignait celui d'enclencher ce processus de réflexivité précisé par Cefaï (2003).

Notre recherche procède d'une socioanthropologie engagée dans les relations à l'autre : nous tentons de comprendre du dedans ce qui s'exprime, en acceptant de participer nous-mêmes à ces expériences. Les entretiens, de plusieurs heures, reconduits jusqu'à trois fois avec un même enquêté, offrent un aller-retour, un jeu de réciprocité dans le donner et le recevoir. Ils sont un espace temporel, spatial, émotionnel... particulier et à inventer à chaque fois. Cet exercice exige de s'affranchir de ses propres valeurs et préjugés, d'interroger ses propres connaissances et limites et d'être ouvert à tous les possibles. Dans ce cadre, l'objectivité s'atteint au travers de cette phase de compréhension de tous les rouages des démarches de chacun, des intentions des uns et des autres, des contextes dans lesquels ils et elles (se) sont engagés. Il s'agit de rendre lisibles, accessibles et compréhensibles les tenants et les aboutissants des actions entreprises au contact de la Nature et de la faune sauvage en particulier.

L'engagement du chercheur est sollicité. Lui reconnaître une "potentialité heuristique » (Le Grand, 2006) apporte un autre regard sur la création d'une connaissance: dans le cadre d'une recherche portant sur un nombre limité d'enquêtés, et sur un thème de recherche suffisamment singulier pour que les «cas » soient peu nombreux, le chercheur devient un témoin privilégié du fait de sa propre expérience ${ }^{4}$. Il peut mesurer son implication, en comprendre le processus tout au long de sa recherche. Il «sait» ce qu'il engage et comment il

\footnotetext{
3 Nous précisons que l'âge ou la situation familiale, par exemple, des enquêtés n'étaient pas ici des critères permettant de justifier des similitudes ou des différences, de dégager une " générabilité interne» (Maxwell, 1999).

4 C'est le cas de l'un d'entre nous (Stéphanie Chanvallon, qui pratique la plongée sous-marine en tant que professionnelle).
}

l'engage. Il peut affiner son analyse parce que son expérience lui offre des clefs, des points de vue, des réflexions et des interrogations, qui peuvent être relativement différents de ceux des enquêtés. Le travail en est peut-être rendu plus difficile et délicat, il tient en alerte, mais élargit les possibilités d'appréhension et de compréhension. La subjectivité agit ici comme « levier et instrument de connaissance » (Lavigne, 2007). Être à la fois à l'intérieur et à l'extérieur: double rôle, double difficulté, double complexité sans doute ; un rendez-vous entre le « eux » et le « je », entre « eux » et « nous », et finalement une autre dimension et une richesse possible.

\section{La Nature comme lieu de reconnexions : les sens dans la Nature}

Se retrouver dans la Nature ne semble pas être sans incidence sur le corps, sur l'être dans son ensemble. Si elle représente un lieu pour se ressourcer ou pour fuir, un lieu de méditation ou un lieu de repos, elle semble agir ici au plus profond de l'être, dans le domaine des perceptions, des sensations, puis des représentations et du vécu. L'expérience sensorielle est parfois volontaire et recherchée (Héas, 2010). La Nature stimule, éveille ou réveille, par le bruit, par les odeurs, par le toucher, par la lumière ou les couleurs. Plus encore, l'expérience de la Nature comblant les sens peut apparaître comme un besoin social, presque une nécessité de détente, de relaxation (Héas, 2004). Elle est en tout cas recherchée comme une quête d'émotions, comme si une alliance évidente se réalisait entre l'Homme et la Nature et reconnectait à l'essentiel. La sensualité participe aussi de ce mode d'expression. M. Zuckerman (cité par André et Baudin, 2002) a développé le concept de recherche de sensations à partir de données psychophysiologiques. Selon lui, la recherche de sensations est implicitement assimilée à une recherche de plaisir et d'activation. D'autres analyses précisent ce ressort de l'action risquée, notamment chez les adolescents et les jeunes adultes (Le Breton, 1991 et 2002). Les activités pratiquées dans la Nature, le simple désir d'y être plongé, sont autant de modalités où les sens et les sensations naissent et prennent forme, répondant à un besoin d'être : « Aujourd'hui, la recherche de sensations est définie comme un trait caractérisé par le besoin d'expériences et de sensations variées, nouvelles et complexes » (André et Baudin, 2002, p. 89), pour répondre à « la susceptibilité à l'ennui », à « l'intolérance à la monotonie, aux activités routinières et répétitives »(ibid.). Dans les sociétés modernes, selon Le Breton (1999), le corps est devenu un élément distinct de la Nature et même dissocié de la nature même de l'Homme. Pourtant, l'être humain semble en recherche de sensations pour retrouver une façon de vivre que nos sociétés ont profondément modifiée. Il ressent probablement un besoin de s'éprouver et de s'épanouir au travers de son corps, dans sa pleine expression, dans des émotions et sensations multiples, 
qu'elles soient liées à des pratiques physiques, à l'art, à la création ou à la rencontre avec l'autre, humain ou non humain (Héas, 2010).

Cette autre perception et conscience du corps permet de redéfinir la conscience de soi, dans son interaction avec le monde, en dépassant au travers d'expériences atypiques et inhabituelles les normes sociales d'un corps dont on ne sait plus s'il est seulement corps-machine ou objet de fantasmes. Quand des femmes ou des hommes partent en quête de la Nature, ils partent aussi en quête d'eux-mêmes. La rencontre avec la Nature dépasse largement le cadre du corps mis en scène et engagé. Cette relation éveille ou réveille un lien profond entre soi et le monde. Pour cet enquêté, naufragé et sauvé - selon ses dires - par deux dauphins ${ }^{5}$, il est primordial de " se sentir vivre et apprendre de la nature, apprendre qui on est. Et cela passe par la compréhension des lois naturelles que nous avons globalement oubliées dans nos sociétés modernes » (2008).

\section{Scission et/ou osmose ?}

Ces pratiques de la Nature se définissent aussi comme des occasions de se mettre en dehors de la civilisation, qui excluent toute forme de «parasitage ». Elles sont censées permettre d'accéder à un état d'osmose avec les éléments naturels. Il s'agit de vivre la Nature en totale liberté, de laisser passer en soi toutes les énergies, les odeurs..., de s'en imprégner comme si l'être devenait un réceptacle, mais aussi un lieu d'échanges (Héas, 2010). Ce sentiment d'osmose procure une nourriture des sens, de véritables «nourritures affectives" (Cyrulnik, 2000). L'osmose est cet état de diffusion de l'un vers l'autre, elle opère un aller-retour, une interpénétration, une influence réciproque entre l'être humain et l'environnement naturel. Pour cet apnéiste de l'extrême, professionnel du no limit, " dès que l'on est sous l'eau, c'est comme si on retrouvait dans cet élément liquide une partie de nous. On épouse les mouvements de la mer, on est confondu au milieu naturel. C'est assez fusionnel. On change de panoplie, on devient un aquarien [...]. Et la palme, c'est comme une prothèse, on s'identifie aux poissons " (2002). L'expression de « l'homme-poisson » (Chanvallon, 2004) acquiert une signification double. « Le poisson est le symbole du contenant redoublé, du contenant contenu [...]. Le poisson est presque toujours significatif d'une réhabilitation des instincts primordiaux » (Durand, 1992, p. 243). Comme le poisson contenant, l'homme, corps réceptacle de sensations, avale par son corps poreux,

\footnotetext{
5 Seul à bord d'un voilier, ce navigateur a vécu un naufrage au large de la Bretagne (bateau retourné). Alors qu'il avait passé plusieurs heures dans l'eau et qu'il se trouvait à bout de forces, deux dauphins seraient apparus et l'auraient maintenu à la surface. Le navire de la SNSM (Société nationale de sauvetage en mer) est arrivé peu après.
}

nourrit son esprit ; comme le poisson contenu, il est luimême avalé par l'eau, la mer qui l'entoure, l'enveloppe, l'englobe, car «l'avalage ne détériore pas, bien souvent même il valorise ou sacralise » (ibid., p. 234).

Cette relation à la Nature est complexe. Dans une précédente recherche autour de l'objet palme, Chanvallon (2004) avait mis en évidence deux approches et deux façons de vivre la plongée. D'un côté, il y a les plongeurs équipés de façon légère (palmes, masque et tuba, voire fine combinaison au plus près du corps), pour qui la pratique est vécue comme la naissance et le berceau de sensations et d'émotions : ressentis tactiles, phénomène de portance, de légèreté, enveloppe liquide protectrice et apaisante. Un sentiment absolu de liberté, de plénitude émerge alors de tout ce qui provient du corps, du cœur et de la sphère cognitive, un tout que l'eau aide à globaliser, à unifier, redessinant l'enveloppe corporelle. Et puis, d'un autre côté, il y a ceux qui s'entourent de mille précautions et objets à la pointe de la technologie. Pour les plus assidus et les plus engagés d'entre eux, la plongée devient même un champ d'expérimentation : de nouveaux mélanges gazeux, de nouveaux instruments de contrôle, des essais, des perfectionnements du matériel, pour des plongées toujours plus profondes, plus longues, plus lointaines. Cela nécessite un grand contrôle des conditions de l'immersion. Au sein de cette mer devenue espace à conquérir, la confiance dans l'outil est indispensable, c'est une cuirasse technologique, comme une scission, à la fois expression de l'intelligence et nécessité pour se protéger d'un milieu tellement hostile. L'Homme essaie et s'essaie, il se mesure dans la démesure, repousse les limites ${ }^{6}$. Durand (1992, p. 227) souligne : " Il existe dans la descente une surdétermination de protections : on se protège pour pénétrer au cœur de l'intimité protectrice [...] l'axe de la descente est un axe intime, fragile et douillet [...]. » Cette immersion, certes surprotégée, est peut-être une inversion de la mort pour une renaissance symbolique. Voilà deux pratiques que tout semble opposer : autant l'une est orientée vers la Nature et pétrie de sensualité, autant l'autre incarne l'esprit conquérant de l'aventurier armé de l'hypertechnologie.

Mais, au-delà de ces apparences, ne s'agit-il pas de la même quête, qui s'accomplit dans la plongée, en empruntant simplement des chemins en apparence différents? On observerait ainsi dans notre culture occidentale, dans notre société patriarcale, à la fois les valeurs - masculines - d'affirmation de soi, de domination, d'expansion, construites sur le modèle rationnel et le réductionnisme, et celles - féminines - de l'intégration, de la préservation, de la coopération, issues de la pensée intuitive et holistique (Capra, 2003). L'opposition

\footnotetext{
6 Les performances en apnée sont, à ce titre, évocatrices : par exemple, 11 minutes 35 secondes est le nouveau record d'apnée statique réalisé par Stéphane Mifsud en juin 2009 (Héas, 2010).
} 
masculin/féminin est-elle si évidente ? Peut-on simplement, comme le proposait Terrasson (1993, p. 145), parler d'une virilité agissante s'opposant à " la vieille équivalence entre nature et principe féminin » ? Ces deux tendances et mouvements n'ont pas à s'opposer, mais à se mutualiser. Dans ce dépassement de soi, dans cette réalisation de soi, pour reprendre les propos de Bergeron (1999), l'individu s'accomplit dans sa propre humanité. Car ce sensible, cette féminité est toujours à l'œuvre, souterraine peut-être, mais en voie d'une nouvelle émergence. L'intimité et le sensible n'appartiennent pas uniquement à des pratiques dites douces et/ou intuitives. Ces deux formes de pratiques liées à la mer en témoignent. À moins que ce dépassement et ce surpassement de soi ne traduisentl'angoisse du temps qui passe ? Nier ce féminin serait alors renoncer à l'identité profonde de tout être. Car ce n'est peut-être pas le féminin qui est refoulé, mais cette féminité, « alliée secrète de la temporalité et de la mort » (Durand, 1992,p. 134). Ainsi, cette quête des sens apparaît comme une quête de sens, dans sa dimension spirituelle, le sens de l'existence, de ce qui échappe et qui est accepté ou refoulé, mais qui est à intégrer quoi qu'il en soit, à un moment ou à un autre, d'une façon ou d'une autre.

\section{Immersions initiatiques}

\section{L'expérience intime comme ouverture}

Les pratiques de la Nature mettent en jeu la notion de profondeur et invitent à s'interroger sur elle. La Nature ne doit pas ici être entendue seulement comme une donnée physique, mais aussi dans sa dimension "géopoétique » (White, 1994). Selon l'auteur, cette dimension renvoieà un espace imprégné des forces du corps et de la pensée, un lieu d'expression des sensibilités et des questionnements existentiels. Ainsi, une plongée en solitaire, une journée passée à gravir un sommet, quelques heures au pied d'un arbre, des semaines à marcher dans un désert, des mois de navigation en haute mer... tout est propice à cette rencontre avec la Nature, qui s'apparente à une rencontre avec soi. Qu'est-ce qui est mis en jeu et investi ? à quel niveau, avec quelle intensité ? et pour quoi ? Alors que certains auteurs voient dans la griserie extrême des activités de pleine nature la reprise en main d'une vie qui échappe, nous pensons qu'il s'agit aussi d'une transformation créatrice de l'être, forme de transcendance, «an exploration of an interior world»(Cantelo, 2003, p. 105), voire un dépassement des peurs.

L'appel de la Nature peut s'inscrire dès l'enfance telle une empreinte - «la simple présence de l'autre nous modifie », précise Cyrulnik (2000) - ou parfois des années après, suivant les circonstances et les événements de la vie. La Nature est une valeur refuge, un lieu de méditation, de ressourcement par rapport à un environnement et une vie sociale trépidante. Ici, la notion de « repos social », développée ailleurs (Héas, 2004), se double du besoin de se retrouver avec soi-même. La solitude est comme un pont, le début d'un long cheminement intérieur entre ce quel'on a été et ce vers quoi on va, le passage presque obligé pour se retrouver soi. La solitude comme une pause nécessaire, un temps pour soi qui ouvre les portes de la réflexion. «On se déshumanise, mais pas dans le mauvais sens, on est juste là, on est ", souligne une navigatrice enquêtée (2007), comme si les conditions de dénuement presque extrêmes, proches des états de survie, permettaient un retour aux sources.

Il y a ici un paradoxe, voire une contradiction entre l'apaisement, d'un côté, et de l'autre, le plein d'énergie qui « booste », ce qu'apportent les pratiques de la Nature. Au-delà des mots, des émotions, c'est un monde empli de valeurs symboliques et d'imaginaire qui sous-tend les propos collectés : " J'avais besoin comme du ventre d'une mère, ce que je trouvais surtout dans la forêt tropicale ", confie (en 2006) ce vétérinaire de formation, réalisateur de films documentaires pour des chaînes télévisées. Le milieu agit ici pleinement : « La forêt est centre d'intimité comme peut l'être la maison, la grotte ou la cathédrale. Le paysage clos de la sylve est constitutif du lieu sacré [...]. Le lieu sacré est une cosmisation [sic], plus large que le microcosme de la demeure, de l'archétype de l'intimité féminoïde» (Durand, 1992, 281). Chacun à sa façon trouve son refuge, en fonction de sa sensibilité particulière, de tout ce qui fait de l'individu un être unique, avec ses aspirations et ses manques.

La fusion avec l'élément naturel est pleinement un vécu que " seuls les initiés peuvent connaître », souligne la navigatrice au long cours (2007). Car « l'initié franchit le rideau de feu qui sépare le profane du sacré ; il passe d'un monde à un autre et subit de ce fait une transformation ; il change de niveau, il devient différent. Initier, c'est introduire » (Chevalier et Gheerbrant, 1982, p. 521). La proximité avec les éléments naturels redonne la plénitude d'être. Comme nous le confiait la navigatrice, « on part avec l'essentiel et on a même l'impression au fur et à mesure de se déshabiller. On retrouve ce qui nous est absolument indispensable. C'est pour ça que je navigue sur des engins relativement simples, petits, basiques, et vraiment on se retrouve dans une espèce de cocon initial »; telle l'image de la coquille de noix que Durand (1992, p. 289) définit comme "l'intimité de l'enceinte coquillaire ». Cependant, la notion même de "cocon initial » fait référence à la chrysalide, à cette transformation en devenir, ce qui est au cœur du voyage de cette enquêtée. Malgré les difficultés et les épreuves du voyage, même si « on en bave vraiment [...], ça avait sa raison d'être! »(2007). Au-delà des motivations premières qui donnent l'énergie et la dynamique nécessaires à l'accomplissement du voyage, semble se profiler une inspiration venue " $\mathrm{du}$ fond de soi », où l'aventure à 
venir devient impérieuse nécessité. Et si prise de risque il y a, elle s'apparente à une force et à une puissance de vie. Pouvoir éprouver ce sentiment « d'être au bon endroit au bon moment » (2007) se vit comme une récompense, une façon de toucher l'absolu parce que le sentiment d'évidence apparaît. Cette évidence s'impose, elle est suffisamment puissante pour impulser de l'énergie dans les périodes de doute. Les enquêtés expriment alors les vocables d' "amour", de "transcendance", de quête «spirituelle». La spiritualité, dans une perspective anthropologique, exprime un rapport au monde propre à soi, ce que souligne entre autres Bergeron (1999).

\section{Connivence}

La quête de la Nature peut être reliée à une quête personnelle particulièrement visible dans la société moderne. C'est ainsi que, décrivant dans la « modernité » une « crise anthropologique » caractérisée par « la dilution des repères et une certaine anomie spirituelle », Soulé et Routier (2008, p. 104) analysent la course au large en solitaire comme un "engagement corporel singulier» (c'est le sous-titre de leur article), à dimension spirituelle. Pour eux, " embarquer » n'est pas seulement synonyme de " coupure spatio-temporelle avec le quotidien », mais aussi de « régénérescence » (ibid., p. 105). De même, pour Bergery et Pech (2002), « larguer les amarres » constitue un acte autant spirituel que matériel. Dans notre travail, nous ne pouvons faire l'impasse sur ce qui anime les enquêtés au-delà des expériences qu'ils relatent : un désir de comprendre, de donner du sens à ce qu'ils vivent dans des situations atypiques, de se dépasser, de dépasser le quotidien pour trouver ou retrouver un « quelque chose » qui les porte plus avant et leur permet sans doute de mieux vivre les difficultés et les épreuves du quotidien. On retrouve la définition de Bergeron (1999, p. 235) de «la vie spirituelle»: l'«entreprise par laquelle la personne humaine tend à unifier son expérience de vie dans l'achèvement et le dépassement de soi-même [...]. On prend la route spirituelle pour s'accomplir dans sa propre humanité ».

Les propos des informateurs précisent ce lien indéfectible à l'Univers. L'enseignant-chercheur enquêté indique (2008) : "Je suis très attaché à cette unité du monde vivant à laquelle j'appartiens et dont tous les hommes font partie. Et cette unité $m$ 'assure une force. » "On se sent appartenir à un Tout, on prend aussi conscience de sa juste mesure ", écrit, de la même manière, Étienne (1999, p. 337), après des années d'explorations scientifiques. Cette représentation n'exclut pas qu'une position particulière soit éventuellement accordée à l'être humain vis-à-vis des autres espèces vivantes. Mais la notion de respect y occupe une place essentielle. Chez les enquêtés, qu'ils soient scientifiques et/ou passionnés de Nature, ce sentiment ressenti d'appartenance à ce «quelque chose » de tellement plus grand que soi devient source d'humilité. La Nature est alors facilement personnifiée dans le discours comme une puissance, inaccessible aux prétentions de l'Homme à vouloir la maîtriser et l'organiser à sa façon. Cela dit, on ne lui prête pas non plus de volonté particulière. Elle est une grandeur et une force à elle seule, mouvante, en évolution permanente ; elle est, tout simplement : "Que nous la regardions, la pensions, l'ignorions, la détruisions, cette terre ne nous appartient pas, elle existe au-delà de nous. Se battre, tomber à genoux, trembler d'incertitude, crier ne sert à rien, qu'à accentuer le silence. Nietzsche disait que la pleine nature n'avait pas d'opinion sur nous, aussi pouvions-nous nous trouver si à l'aise en son sein » (Ink, 1994, p. 338).

Cette forme nouvelle de spiritualité, qui vise à mettre les humains "en syntonie avec l'univers", (Bergeron, 1999) se retrouve chez des scientifiques d'horizons différents : astronomes, physiciens, biologistes (Laszlo, 2005 ; Capra, 2003 ; Costa, 2005). Elle fait figure de défi dans la mesure où elle met en évidence la nécessité de penser autrement le monde et notre relation au monde.

\section{De l'extraordinaire à l'évidence}

\section{De la sensibilité naturelle à la « stratégie » professionnelle}

«Opposer rationnel et irrationnel nous apparaît particulièrement absurde, car nous ne jugeons irrationnel que ce dont nous ignorons encore les lois » (Henri Laborit, cité par Meinard [1998, p. 11]). Cette façon de considérer l'irrationnel offre une perspective pour une exploration et une compréhension des relations des humains à la Nature, si on l'applique à la façon dont nos témoins privilégiés vivent les leurs, aux émotions qu'ils disent en tirer, aux valeurs qu'ils y mettent et aux significations qu'ils donnent à leurs expériences. L'irrationnel, selon Laborit (1974, p. 95), constitue la source profonde de notre créativité, il est "le trésor caché des intuitions générales et des motivations qui les engendrent». Pourtant, ce qui paraît irrationnel déroute, crée le doute, remet en question ; et, devant l'inconnu, il est difficile de ne pas se raccrocher à ce que l'on connaît pour se rassurer sur sa propre expérience de la connaissance et sur les valeurs que l'on porte. Ainsi, pour Varvoglis (1992), le refus du nouveau par attachement à l'ancien crée ce qu'il nomme, après d'autres ${ }^{7}$, une "dissonance cognitive » difficile à assumer. "Aussi, nous avons tendance à sélectionner les informations qui correspondent à nos croyances, évitant ainsi de nous retrouver en porte-à-faux. Lorsque nous ne pouvons plus ignorer l'information

\footnotetext{
7 Leon Festinger notamment, dès 1957, dans une théorie célèbre.
} 
conflictuelle, nous nous attachons à la rationaliser et à supprimer son sens premier » (ibid., p. 170).

Mais, sans tourner le dos à la modernité et à ses acquis scientifiques, nous pouvons faire référence à ces situations proches du paranormal, ou phénomènes «psi » : la définition la plus souvent utilisée dans la littérature scientifique est celle d'Alcock (1981), pour qui « un phénomène paranormal est un phénomène qui est inexplicable dans les termes de la science actuelle ». Les phénomènes «psi » peuvent être définis comme objectivement inexplicables (ils sont quasi impossibles physiquement parlant), mais subjectivement compréhensibles : ils sont chargés de sens pour la ou les personnes concernées, au niveau conscient ou, plus souvent, au niveau inconscient. Comment nos enquêtés expliquentils leurs expériences, leur cheminement, et quel sens leur donnent-ils? Comment comprendre la perception humaine de l'environnement et du monde vivant en tant que mode de communication qui sort de l'ordinaire? Pour cet apnéiste chasseur et vidéaste animalier professionnel, « cen'est pas rationnel du tout, mais très souvent, juste par le fait de se mettre dans l'eau, de regarder vers le fond, on sait immédiatement, tu le sens, il y a des choses... On sent le poisson. Est-ce que l'on a réussi à compiler toutes sortes d'informations dont certaines qui nous échapperaient? De façon inconsciente, on développe une sorte de sixième sens ? Oui, effectivement il y a des choses qu'on sait, que l'on sent avant qu'elles n'arrivent ». L'intuition, ce fait de « sentir venir les choses » qui serait la connexion en une fraction de secondes de connaissances et d'expériences variées (Gladwell, 2006), peut s'avérer fort utile dans le domaine professionnel, entre autres. Si nous prenons l'exemple des professionnels de la mer, ces hommes et ces femmes apprennent à travailler avec les éléments naturels, et pouvoir anticiper sur des phénomènes leur permet de mieux gérer leur activité. Il en va ainsi de cet enseignant-chercheur, spécialiste du plancton, dont les micro-organismes toxiques : «Je pense qu'il y a des signes avant-coureurs et on est tout doucement en train de les mettre en évidence. Mais, pour montrer ça, il faut observer en permanence l'eau [...]. L'idée, c'est de pouvoir sentir le milieu, parce que dans le milieu il y a des signaux d'alarme [...] le professionnel, s'il sent le milieu, eh bien, il va changer son comportement, il a le temps de mettre une stratégie en place. On peut, quinze jours avant, sentir le vent venir et s'adapter. M. [collègue de travail] sait déjà sur la route en venant, avant de faire ses prélèvements. Elle a une sensibilité... » (2006). Dans le discours et les pratiques, il y a ce qui relève des données scientifiques et puis tout ce qui est en aval, ce qui naît du ressenti, des impressions, de la réflexion, mais dont on oublie aussi le caractère premier lorsque la démarche scientifique a été conduite dans les règles de l'art et a apporté la confirmation. Ce chercheur revendique cette capacité à anticiper, à ressentir, par le « flair ».
Dans son expérience professionnelle, il est confronté à ce refus des " autorités » scientifiques d'entendre ce qui est prouvé autrement que par des instruments de mesure conformes : "La pifométrie c'est une science ; l'à-peuprès, c'est une science. On se retrouve avec des gens qui ont été formatés scientifiques purs et qui te disent : "Non, tant que tu ne peux pas le prouver, tu ne peux pas le dire." Ça, c'est catastrophique parce que, quand on a la preuve, c'est trop tard et on a bonne mine ! Il faut que les ressentis rentrent en ligne de compte ! » Travailler avec un élément naturel développe une attention particulière à ses évolutions, à la lecture de ses changements. La nécessité et les impératifs professionnels obligent quelque part à cette sensibilité.

\section{Traduire l'irrationnel en action}

Ce qui est vécu et perçu, exprimé sous le vocable du " sentir, ressentir », se manifeste avec intensité parmi les enquêtés. "C'est» ou bien « je sens » sont des affirmations qui s'imposent tout simplement parce qu'elles sont vécues de l'intérieur, avec intensité. Elles font appel à un ensemble de prédispositions et dispositions : ouverture, lâcher-prise, réceptivité, expérience, état d'esprit et état de conscience particuliers, harmonie avec le milieu. Communiquer ou communier avec la Nature nécessite, comme toute démarche volontaire, un engagement, si ce n'est un désir profond. Pourtant, les expériences des enquêtés ne sont pas toujours consécutives à une action réfléchie et décidée, tout particulièrement dans la rencontre avec un animal sauvage. Parfois, des situations se vivent dans l'imprévu, ou relèvent "presque du hasard», comme aiment le souligner avec ironie plusieurs des enquêtés pour qui il n'y a pas vraiment de hasard. En effet, selon eux, chaque rencontre, chaque événement est à sa juste place. Ces situations engendrent pour eux de nouvelles perspectives et visions dans leur relation au monde.

Ces expériences sont ressenties comme particulières, notamment dans la mesure où elles circulent par des voies de communication autres que la parole. C'est un état d'esprit et un état d'être au monde qui préparent ou favorisent des rencontres attendues, suscitées ou imprévues avec des animaux sauvages, des conditions écologiques extrêmes, exceptionnelles. Ceux qui les expérimentent les relatent a posteriori comme naturelles, évidentes même. Pour autant, de quelles évidences s'agit-il aux yeux des enquêtés?

Dans le cadre de son expérience personnelle et professionnelle comme plongeuse, chercheur et réalisatrice de films documentaires animaliers, l'une d'entre nous (S. Chanvallon) a expérimenté ce sentiment d'évidence. Elle a eu maintes occasions de faire des rencontres avec la faune sauvage aquatique, mais en 2001, ainsi qu'elle l'a écrit dans son carnet de bord, elle a vécu l'une d'elles 
comme une expérience "exceptionnelle», comme un « un temps fort dans [sa] vie », car tout dans le comportement de l'animal (il s'agissait d'une raie manta, Manta birostris) lui donnait à penser qu'il était littéralement venu la chercher, l'avait conduite à l'écart du groupe des plongeurs et des autres raies et lui avait permis de rester suspendue sous lui pendant toute une plongée. C'est alors que s'impose le sentiment $d^{\prime}$ «avoir touché à l'essentiel » et qu' « en même temps, [c'est] tellement évident, simple». Cette expérience parachevait un ensemble de ressentis et d'émotions de la journée et en constituait comme une acmé. Ce genre de témoignage se retrouve dans les propos d'enquêtés relatant certaines rencontres avec des orques (Orcinus orca) ou des dauphins (Tursiops truncatus), par exemple. Cette relation est vécue comme une relation avec la Nature et, plus fondamentalement, comme l'évidence d'une communication avec tout ce qui vit ${ }^{8}$.

Suivant la même logique, les représentations communes de la Nature exprimées par les enquêtés sont enveloppées dans un discours sur l'expérience vécue où l'on retrouve des modes de perception et de communication inhabituels. S'y traduit un sens particulier du ressenti de l'autre, du ressenti à distance. Cela résulte probablement d'un contexte spécifique qui provoque la mise en place progressive d'un état d'esprit contemplatif, d'une faculté exceptionnelle d'être réceptif et en phase avec le milieu. Parler de « ressenti » veut dire ou bien que ce qui est vécu ne peut pas transiter par d'autres sens, ou bien que cette capacité de ressentir est favorisée par un contexte particulier producteur à la fois de détachement (par rapport aux aspects matériels et contraignants de l'existence) et d'un lien avec les énergies de la vie (et avec des éléments sans doute plus subtils, tels la beauté et les sentiments qu'elle éveille). Sans doute est-il également nécessaire, comme préalable, que l'individu soit prêt à ce type d'expérience, qu'il soit disponible, ouvert à tout possible et dégagé de tout environnement susceptible de « brouiller » les échanges. Ce que nous observons chez les enquêtés, ce sont des situations qui laissent à penser que l'individu se met progressivement dans un état modifié de conscience, loin du stress qui bloque une « conscience plus subtile » (Varvoglis, 1992 ; Héas, 1996), de façon à être en interconnexion avec le monde environnant ${ }^{9}$.

$\mathrm{Si}$, d'une façon générale, les rencontres avec la Nature offrent le sentiment de communion, d'un vécu intime particulier, les rencontres avec les animaux promettent une autre aventure. La Nature, c'est alors l'Autre non humain, cette autre intelligence. Et cette rencontre avec l'animal sauvage, ces "animaux singuliers » (Lestel,

\footnotetext{
8 Peut-être, d'ailleurs, que cette expérience sensible est vécue également dans un quotidien avec les animaux dits de compagnie, sans que celle-ci soit suffisamment explorée. En effet, le risque est important d'occulter la réalité de certains faits parce qu'ils sont imprégnés du quotidien.
}

2007), espérée ou fortuite, souvent bouleversante, est porteuse de sens et motivée par les deux protagonistes : l'animal et l'homme ou la femme. Car cette rencontre, en particulier en mer, est bien décidée par l'animal qui accepte d'entrer en contact, comme on vient de le voir. L'échange se fait alors sur un mode nouveau, dans une communication non verbale, « on est sur la même longueur d'onde ", voire dans cette " chambre secrète de la communication », entre sympathie, empathie, télépathie, précisent les enquêtés. Pour Cyrulnik (2000), il s'agit parfois entre l'animal et l'Homme d'un « ensorcellement réciproque ». Et la transformation intérieure réside justement dans ce que l'Autre renvoie par sa différence, parce qu'il vient bousculer des repères bien établis. Cela passe par l'exploration, la comparaison, le rapprochement ou la distanciation, tout ce qui permet de vivre et d'éprouver sa différence apparente, et de renforcer sa propre consistance.

En acceptant de voir l'animal différemment, les enquêtés découvrent du nouveau. Pour cette exchampionne du monde de planche à voile qui a, entre autres, rencontré les dauphins, " tous les animaux sont une clef pour nous ouvrir à une qualité intérieure » (2009). Pour ce moniteur de plongée, à travers le contact avec l'animal sauvage, ce qui est en jeu, c'est «élargir sa conscience de la nature ou sa spiritualité » (2008). Quant à cet apnéiste qui a nagé avec les orques, il affirme (2009) : «Ah oui! j'ai grandi. Cette expérience-là m'a enrichi énormément. Après, tu perçois les choses qui t'entourent avec un autre regard. Ces expériences-là, elles ne modifient pas que ton échelle de valeurs, mais aussi ton fonctionnement [...]. Être centré sur ce que l'on est, pas seulement sur son ego. »

L'Autre est celui qui permet de dépasser ses représentations, ses schémas de pensée imprégnés de tant de rationalité, pour découvrir son moi, au-delà de son inscription dans l'espace et le temps de la société. L'expérience n'est pas incorporelle, elle est émotion, paroxystique parfois, dans toutes les dimensions de l'être et elle modifie profondément sa façon de penser le monde et de s'y inscrire. Pour Winch (cité par Cefaï, 2003,pp. 234 et 257), « la capacité de saisir le sens de la vie ne dépend pas du seul individu concerné », mais « dépend aussi des possibilités que la culture dans laquelle il vit offre, ou n'offre pas, de produire un tel sens ». Certes, mais ne faut-il pas aller plus loin? Un individu peut être suffisamment autonome pour aller chercher dans une autre source que celle de la

\footnotetext{
9 Varvoglis (1992, p. 243) considère que les phénomènes «psi » agissent avec une certaine spécificité : «[...] en effet, l'agent se trouve dans une relation particulière avec un événement, ou une personne, et non pas avec le monde entier, ou le Tout [...]. Bien que toutes les facettes de l'Univers puissent être interreliées, il faut considérer que certaines relations sont primordiales par rapport à d'autres et qu'elles dénotent un plus haut niveau d'intimité. »
} 
culture dans laquelle il vit ce qui peut l'aider à donner du sens à son expérience et à son parcours personnel ou/et professionnel. L'idée de l'expérience intime, profonde, dépasse la notion de culture, elle se place quelque part en deçà parce qu'elle est une rencontre avec Soi (Jung, 1987). Le « je » ne traduit peut-être pas, ou plus, cet individualisme replié sur soi, mais un «je» qui s'affirme et s'apprête à rencontrer l'Autre, profondément, inévitablement, dans un duo qui saura être le terrain vers de nouvelles remises en cause et de nouveaux questionnements.

\section{Conclusion}

Seule une conceptualisation plastique et multiniveaux (Gonzalez, 2009) peut permettre d'appréhender les relations de l'Homme à la Nature, de tenter de comprendre ces «singularités plurielles» (Bouvier, 1997). Récits de rencontres, regards sur la vie, représentations, engagements, sensibilités, les acteurs rencontrés sont passionnés et se sentent partie intégrante de la Nature. C'est le cas notamment d'une minorité d'individus, du « cercle très fermé » de ceux qui nagent avec les plus grands prédateurs des mers ou des navigateurs au long cours. "Ces minorités ne peuvent imposer leurs solutions, mais aider les gens à trouver leurs propres solutions en activant leur imagination et leur affectif » (Moscovici, 2002, p. 140). Et $c^{\prime}$ est cela qui importe : comment, ici ou ailleurs, imaginer des principes de vie, enclencher des pratiques ? Il ne s'agit pas d'envisager un « écocitoyen », car ce serait à nouveau tomber dans le piège d'une distinction sociopolitique, mais tout simplement un citoyen, un habitant de la Terre qui percevrait autrement et par lui-même sa relation à son environnement, à la Nature, à l'Univers. Il n'agirait pas uniquement par intérêt pour les générations futures, sa descendance, ou par bonne conscience, mais tout simplement par compréhension des lois de la Nature, des principes d'organisation et d'évolution des communautés écologiques, de leurs réseaux d'interdépendances, de tout ce dont il fait partie (Naess, 2004 ; Capra, 2003).

La Nature n'est pas uniquement cette nature que l'on recrée en ersatz, ni un objet de science qui se fragmente en divers sujets, elle n'est pas que cet environnement instrumentalisé au cœur des tensions politiques locales et internationales. La Nature permet à l'Homme d'accéder à d'autres dimensions de son être. «[...] profond - écrit Séjournant (2001, p. 295) - est notre besoin d'écologie personnelle - l'anthropologue Gregory Bateson parlait d'une " écologie de l'esprit ". Un déséquilibre intérieur important serait un handicap trop grave, dans la démarche d'un écologiste, ou d'un militant humanitaire conséquent. Comment pourrais-je défendre l'Humanité et la Terre-Mère si je ne les ai pas d'abord retrouvées en moi ? » Évoquant tant la pollution «dure » (les innombrables déchets) que la pollution «douce» (celle, plus insidieuse, du monde de l'image envahissant le quoti- dien), Serres (2008) en appelle à une nécessaire « dépossession du monde » pour (re)découvrir la beauté de la Nature. Sans doute. Mais ce n'est pas là qu'une exigence collective, elle est aussi individuelle et personnelle. Si « dépossession du monde » il doit y avoir, elle suppose une repossession de soi la portant.

Les exemples fournis par les enquêtés illustrent que des voies allant dans ce sens existent. Ils montrent que des pratiques impliquant un fort contact avec des éléments naturels peuvent être vécues comme une expérience d'une nature ressentie comme telle, et que cette expérience est source d'une redécouverte de soi. Les enquêtés sont des aventuriers, oui, mais avant tout des aventuriers d'eux-mêmes. Ils et elles ont pensé et vécu la Nature comme un guide et non comme une finalité de l'action. Leur expérience initiatique a été le point de départ d'une dynamique d'évolution dans leurs conceptions du monde, de soi, de l'Autre, du sens donné à ces relations, pour expérimenter du nouveau et aller fouiller dans cet « irrationnel qui ne paraît riche que de rationalité potentielle » (Laborit, cité par Meinard [1998, p. 11)].

De ce point de vue, l'expérience de la Nature fait partie de ces « échappées belles » qui, à l'instar de la création artistique, nous ressourcent en nous plongeant dans les forces instinctives, pour oser l'inconnu comme un défi à l'ordre institué, transgresser, se dépasser, créer dans un nouvel élan. Selon Ormiston (2003), il ne s'agit pas de rechercher une transformation révolutionnaire $\mathrm{du}$ monde, dans une utopie écologique, mais de comprendre comment l'individualisme moderne exprime un renouvellement des relations à la Nature. En quoi la société peut-elle alors être affectée en retour ? Quelles réflexions sur la place publique sont alors possibles? Comment faire en sorte de penser et d'agir dans une éthique qui reconnaîtrait profondément la valeur intrinsèque de tout existant, simplement par respect pour ce qui est, et enfin sortir de l'exclusivité humaine (Bailly, 2007) ?

\section{Références}

Alcock, J.E., 1981. Parapsychology, Science or Magic? A Psychological Perspective, Oxford and New York, Pergamon Press.

André, J., Baudin, M., 2002. La Vie sensorielle: la clinique à l'épreuve des sens, Paris, PUF.

Bailly, J.-C., 2007. Le Versant animal, Paris, Bayard.

Bergeron, R., 1999. Pour une spiritualité du troisième millénaire, Religiologiques, 20, 231-246.

Bergery, L., Pech, A., 2002. Les raisons du succès de la voile, Espaces, 198, 50-54.

Béteille, R., 1996. Le Tourisme vert, Paris, PUF.

Bouvier P., 1997. L'objet de la socio-anthropologie: crise, déstructuration, recomposition, perdurance, SocioAnthropologie, 1 (mis en ligne le 15 janvier 2003 : http:// socio-anthropologie.revues.org/index27.html).

Cantelo, B., 2003. Art as a way of knowledge, Ethnologies, 25, 1, 87-107. 
Capra, F., 2003. La Toile de la vie: une nouvelle interprétation scientifique des systèmes vivants, Paris, Éditions du Rocher.

Cefaï, D., 2003. L'Enquête de terrain en sciences sociales, Paris, La Découverte.

Chanvallon, S., 2004. De l'Homme et de l'eau : la palme de plongée comme analyseur et révélateur des rapports de l'homme au milieu aquatique. Approche sociohistorique et anthropologique. Mémoire de DEA, Université Rennes 2, Rennes.

Chevalier, J., Gheerbrandt A. (Eds), 1982. Dictionnaire des symboles, Paris, Robert Laffont.

Corneloup, J. (Ed.), 2007. Sciences sociales et loisirs sportifs de nature, L'Argentière-la-Bessée, Éditions du Fournel.

Costa, J.-P., 2005. Entretien avec Jean-Patrick Costa sur le chamanisme, par Bertrand Méheust, Synapse, 216. Disponible sur: http://www.metapsychique.org/Entretien-avec-JeanPatrick-Costa.html.

Cuche, D., 2004. La Notion de culture dans les sciences sociales, Paris, La Découverte.

Cyrulnik, B., 2000. Les Nourritures affectives, Paris, Odile Jacob.

Durand, G., 1992. Les Structures anthropologiques de l'imaginaire, Paris, Dunod.

Étienne, J.-L., 1999. Le Pôle intérieur: mener sa vie comme une aventure, Paris, Hoëbeke.

Fromm, E., 1975. La Passion de détruire : anatomie de la destructivité humaine, Paris, Robert Laffont.

Geertz,C., 1986. Savoir local, savoir global : les lieux du savoir, Paris, PUF.

Gillet, J.-M., Sorzana, B., 2008. Les Politiques sportives au défi du développement durable, Voiron, Territorial éditions.

Gladwell, M., 2006. La Force de l'intuition, Paris, Robert Laffont.

Gonzalez J., 2009, Ontonlogie des plantes psychoactives dites « de pouvoir » et états modifiés de conscience, in Baud, S., Midol, N. (Eds), La Conscience dans tous ses états. Approches anthropologiques et psychiatriques: cultures et thérapies, Issyles-Moulineaux, Elsevier Masson.

Gould, S.J., 2001. Les Coquillages de Léonard: réflexions sur l'histoire naturelle, Paris, Le Seuil.

Héas, S., 1996. La Relaxation comme "médecine » de ville?. Thèse de sociologie, Université Strasbourg 2, Strasbourg.

Héas, S., 2004. Anthropologie des relaxations : des moyens de loisirs, de soin et de gestion personnelle?, Paris, L'Harmattan.

Héas, S., 2010. Les Virtuoses du corps: enquête auprès d'êtres exceptionnels, Paris, Max Milo.

Héas, S., Bodin, D., Robène, L., Jude, A., 2006. Football féminin : un exotisme genré ? Étude de cas d'une association locale, Africa Development, XXXI, 1, 150-167.

Ink, L., 1994. Il suffit d'y croire, Paris, Robert Laffont.

Jung, C.G., 1987 [nouv. éd.]. L'Homme à la découverte de son âme : structure et fonctionnement de l'inconscient, Paris, Albin Michel.

Laborit, H., 1974. La Nouvelle Grille, Paris, Robert Laffont.

Laszlo, E., 2005. Science et champ akashique, Outremont (Québec), Ariane Éditions.

Lavigne, C., 2007. À qui appartient l'objet de recherche ? Penser l'implication du chercheur dans son objet: le handicap (surdité), Nouvelle revue de psychosociologie, 2, 4, 25-39.
Le Breton, D., 1991. Passions du risque, Paris, Métailié.

Le Breton, D., 1999. L'Adieu au corps, Paris, Métailié.

Le Breton, D., 2002. Conduites à risque : des jeux de mort au jeu de viver, Paris, PUF.

Le Grand, J.-L., 2006. Implexité : implications et complexité (http:/ / www.barbier-rd.nom.fr/JLLeGrandImplexite.html).

Lebreton, F., Héas, S., 2007. Regard ethnographique sur une communauté secrète: la spéléologie urbaine, Ethnologie française, XXXVII, 2, 345-352.

Lebreton, F., Héas, S., Bodin, D., Robène, L., Aït Abdelmalek, A., 2008. Terre et ciel: étude sociologique d'espace-temps sportifs marginaux, Espaces et sociétés, 132-133, 209-222.

Le Hénaff, Y.,Héas, S., 2007. Tatouages et cicatrices : décors sportifs, Paris, L'Harmattan.

Lestel, D., 2007. L'Animalité : essai sur le statut de l'humain, Paris, L'Herne.

Lussault, M., 2003. Nature, in Lévy, J., Lussault, M. (Eds), Dictionnaire de la géographie et de l'espace des sociétés, Paris, Belin. Article mis en ligne le 18 mars 2003 sur EspacesTemps.net : http://www.espacestemps.net/document404.html.

Mauss, M., 2007. Essai sur le don : forme et raison de l'échange dans les sociétés archaïques, Paris, PUF.

Maxwell, J.A. 1999. La Modélisation de la recherche qualitative : une approche interactive, Fribourg, Éditions universitaires de Fribourg.

Meinrad, H., 1998. La Rationalité d'un discours africain sur les phénomènes paranormaux, Paris, L'Harmattan.

Moscovici, S., 2002. De la nature pour penser l'écologie, Paris, Métailié.

Naess, A.,2004. Qu'est-ce quel'écologie profonde ?,L'Écologiste, 12, 40-47 [texte traduit de Deep Ecology for the 21st Century].

Ormiston, A., 2003. Hegel on "Negotiating Transcendence", Ethnologies, 25, 1, 55-86.

Rossi, I., Kaech, F., Foley, R.-A., Papadaniel, Y., 2008. L'éthique à l'épreuve d'une anthropologie en milieu palliatif: de l'insertion à la restitution, ethnographiques.org, 17 (http:// www.ethnographiques.org/2008/Rossi,et-al).

Séjournant, M., 2001. Le Cercle de vie : initiation chamanique d'une psychothérapeute, Paris, Albin Michel.

Serres, M., 2008. Le Mal propre : polluer pour s'approprier ?, Paris, Le Pommier.

Soulé, B., Routier, G., 2008. La course au large en solitaire: regards sociologiques sur un engagement corporel singulier, in Ferréol, G., Loisirs, sports et sociétés, Besançon, Presses universitaires de Franche-Comté, 99-110.

Terrasson, F., 1993. La Peur de la nature, Paris, Sang de la terre.

Varvoglis, M., avec la collaboration de Hardy, C., 1992. La Rationalité de l'irrationnel : une introduction à la parapsychologie scientifique, Paris, InterÉditions.

White, K., 1994. Le Plateau de l'albatros: introduction à la géopoétique, Paris, Grasset.

Wieviorka, M (Ed.), 2007. Les Sciences sociales en mutation, Auxerre, Éditions Sciences humaines.

Reçu le 8 mars 2008. Accepté le 3 mars 2011. 